\title{
Individualized dosing guidelines for PEGasparaginase and factors influencing the clearance: a population pharmacokinetic model
}

Haematologica 2021

Volume 106(5):1254-1261

\section{Correspondence:}

INGE M. VAN DER SLUIS

i.m.vandersluis@prinsesmaximacentrum.nl

Received: November 4, 2019.

Accepted: April 9, 2020.

Pre-published: April 23, 2020.

https://doi.org/10.3324/haematol.2019.242289

(C)2021 Ferrata Storti Foundation

Material published in Haematologica is covered by copyright. All rights are reserved to the Ferrata Storti Foundation. Use of published material is allowed under the following terms and conditions:

https://creativecommons.org/licenses/by-nc/4.0/legalcode. Copies of published material are allowed for personal or internal use. Sharing published material for non-commercial purposes is subject to the following conditions:

https://creativecommons.org/licenses/by-nc/4.0/legalcode, sect. 3. Reproducing and sharing published material for commercial purposes is not allowed without permission in writing from the publisher.

\author{
Robin Q.H. Kloos, ${ }^{1}$ Ron Mathôt, ${ }^{2}$ Rob Pieters ${ }^{3}$ and Inge M. van der Sluis ${ }^{1,3}$ \\ ${ }^{1}$ Department of Pediatric Oncology and Hematology, Sophia Children's Hospital - \\ Erasmus MC, Rotterdam; ${ }^{2}$ Department of Hospital Pharmacy, Amsterdam University \\ Medical Center, University of Amsterdam, Amsterdam and ${ }^{3}$ Princess Máxima Center for \\ Pediatric Oncology, Utrecht, the Netherlands
}

\section{ABSTRACT}

onsiderable inter- and intra-patient variability exist in serum activity levels of PEGasparaginase, essential for pediatric acute lymphoblastic leukemia (ALL) treatment. A population pharmacokinetic (popPK) model was developed, identifying patient characteristics that explain these variabilities. Patients $(n=92)$ were treated according to the Dutch Childhood Oncology Group (DCOG) ALL-11 protocol, using therapeutic drug monitoring to individualize PEGasparaginase doses. Non-linear mixed effects modeling (NONMEM) was used to analyze popPK evaluating several covariates. The final model was validated using an independent database $(n=28)$. Guidelines for starting doses and dose adjustments were developed. A one-compartment model with timedependent clearance was adequately described in popPK. Normalization of clearance and volume of distribution by body surface area reduced inter-individual variability. Clearance was $0.084 \mathrm{~L} /$ day $/ \mathrm{m}^{2}$ for 12.7 days, increasing by $0.082 \mathrm{~L} /$ day $/ \mathrm{m}^{2} /$ day thereafter. Clearance was $38 \%$ higher during an infection, and $11-19 \%$ higher during induction treatment than during intensification and maintenance $(P<0.001)$. In order to target an asparaginase activity level of $100 \mathrm{IU} / \mathrm{L}$, a loading dose of $800 \mathrm{IU} / \mathrm{m}^{2}$ (induction) and $600 \mathrm{IU} / \mathrm{m}^{2}$ (intensification) is advised. In conclusion, variability of PEGasparaginase activity levels can be explained by body surface area, the treatment phase and the occurrence of an infection. With this popPK model, PEGasparaginase treatment can be individualized further, taking into account the covariates and the dosing guidelines provided. (clinicaltrials gov. Identifier [CCMO register]: NL50250.078.14).

\section{Introduction}

Asparaginase plays an important role in the treatment of pediatric acute lymphoblastic leukemia (ALL). The drug converts asparagine in aspartic acid and ammonia, resulting in apoptosis of the leukemic cells, as these cells highly depend on extracellular asparagine pools for protein synthesis. ${ }^{1-3}$ An asparaginase activity level of $>100 \mathrm{IU} / \mathrm{L}$ is considered to be sufficient for complete asparagine depletion. ${ }^{4-10}$ Currently, different formulations of asparaginase derived from Erwinia chrysanthemi or Escherichia coli (E. coli) are available in clinical practice, all with different pharmacokinetic (PK) properties. ${ }^{11,12}$ One of these formulations is the polyethylene glycol (PEG) conjugated form of E. coli asparaginase, also known as PEGasparaginase, which has several advantages compared to the native E.coli asparaginase. First, the risk of developing a neutralizing hypersensitivity reaction to asparaginase is reduced when using PEGasparaginase. ${ }^{13-15}$ Second, PEGasparaginase can be administered less frequently than other asparaginase formulations because of its relatively low clearance (CL) ${ }^{16,17}$ Therefore, PEGasparaginase is currently used as a first-line formulation in most developed countries. However, considerable inter- and intrapatient variability of PEGasparaginase levels has been observed and it is, therefore, difficult to determine the right dose for an individual patient. ${ }^{17,18}$ Currently, asparaginase therapy in Dutch pediatric patients with ALL, treated according to the Dutch Childhood Oncology Group (DCOG) ALL-11 protocol, is individualized with ther- 
apeutic drug monitoring (TDM). However, more insight into PEGasparaginase population (popPK) - and especially in characteristics explaining the variability - is needed to optimize individualized dosing. Recently, Hempel et al. described the body surface area (BSA) as an important factor explaining variability in CL. However, the influence of other patient factors is still unknown. ${ }^{17}$ Therefore, the aim of this study was to describe the PK of PEGasparaginase in our cohort, to gain more insight into factors influencing the CL, and to develop a dosing guideline for PEGasparaginase therapy in children.

\section{Methods}

\section{Patients and treatment protocol}

Patients (1-18 years old) with newly diagnosed ALL between November 2014 and May 2017, treated according to the ALL-11 protocol in the Sophia Children's Hospital-Erasmus MC, Rotterdam, were included. In these patients trough, top, week and other levels were prospectively measured. In addition, asparaginase levels measured after the last dose, as part of the TDM program from patients from other Dutch pediatric oncology centers, were included. The study was approved by the Institutional Review Board (clinicaltrials gov. Identifier [CCMO register]: NL50250.078.14). Informed consent was obtained from patients $\geq 12$ and the parents in accordance with the Declaration of Helsinki.

Patients were stratified as standard (SR), medium (MR) and high risk (HR) after induction. Online Supplementary Table S1 describes the complete treatment for each risk group. Figure 1 shows the concomitant chemotherapy per treatment phase. All patients were treated with three doses of PEGasparaginase $\left(1,500 \mathrm{IU} / \mathrm{m}^{2}\right.$, biweekly) during induction (protocol $1 \mathrm{~A}$ and $\left.\mathrm{B}\right)$. For SR and MR patients, the subsequent dose(s) were individualized based on trough asparaginase activity levels (Online Supplementary Table S3). SR patients received one individualized dose after an interval of approximately 12 weeks (protocol IV); MR patients received 14 individualized doses, either directly after the first three doses or after an interval of approximately 12 weeks during intensification and maintenance as part of a randomized study. HR patients received another two to five doses with a fixed dose of $1,500 \mathrm{IU} / \mathrm{m}^{2}$. Patients were switched to Erwinia asparaginase in case of a neutralizing allergy or silent inactivation. PEGasparaginase was administered intravenously over 1 hour.

\section{Measurements}

Measurements of asparaginase levels and antibodies are described in the Online Supplementary Appendix.

\section{Population pharmacokinetic analysis}

The popPK were analyzed using non-linear mixed models (see the Online Supplementary Appendix). Several structural models were evaluated using one and two compartment models with linear, nonlinear, and time-dependent elimination. Since BSA is known to highly influence the PK of PEGasparaginase, BSA (calculated with the Mosteller formula ${ }^{19}$ ) was included in the structural model to scale the volume of distribution $(\mathrm{Vd})$ and $\mathrm{CL}$.

\section{Covariate analysis}

Several demographic, clinical and therapy-related covariates were evaluated (Online Supplementary Table S2). Infections were defined as fever $\left(>38^{\circ} \mathrm{C}\right)$ and hospital admission or prescription of antibiotics. Beside differences in the treatment phase and con- comitant chemotherapy, several other supportive care drugs were evaluated. First, the covariates were explored with univariate analyses after which significant covariates $(P<0.05)$ were evaluated using a stepwise forward inclusion, followed by backward elimination $(P<0.001)$ in a multivariate analysis.

\section{Model validation and development of dosing guidelines}

The final model was validated using goodness of fit plots and visual predictive checks (VPC), see the Online Supplementary Appendix. An independent database, which was obtained by randomly selecting $25 \%$ of the population, was used for the external validation of the model.

In order to develop dosing guidelines, Monte Carlo simulations were performed. Starting doses were calculated targeting trough asparaginase activity levels $>100 \mathrm{IU} / \mathrm{L},>250 \mathrm{IU} / \mathrm{L}$ and $>350$ IU/L, taking into account the significant covariates.

Dosing guidelines were developed targeting a trough asparaginase activity level of $100-250 \mathrm{IU} / \mathrm{L}$ or $250-400 \mathrm{IU} / \mathrm{L}$ based on either week levels or trough levels.

\section{Results}

\section{Patients and samples}

In total, 120 patients were included in the study. Ninety-two patients were included in the index dataset and 28 patients in the validation dataset. Online Supplementary Table S2 describes the patient characteristics of the two datasets. The patients in the validation database were older (median 8.0 years, interquartile range (IQR), 3.3-12.5 years) than in the main database (median 4.8 years, IOR, 3.3-8.2 years), and had a higher weight (median $28.0 \mathrm{~kg}, \mathrm{IOR}, 16.6-47.9 \mathrm{~kg}$ vs. $19.2 \mathrm{~kg}$, IOR, 14.9$29.3 \mathrm{~kg})$.

Table 1 shows the characteristics and distribution of the samples. In the index database, 816 samples were measured in 92 patients. The majority of the levels were top, week or trough levels, and were measured during the intensification and maintenance phase of the MR group. Figure 2 shows all asparaginase activity levels plotted against the time after dose.

\section{Structural model}

The development of the structural PK model is described in the Online Supplementary Results. The estimated popPK parameters are stated in Table 2. Concentration-time profiles were best described by a one-compartment model. Adding a second compartment did not improve the model. Normalization of the CL by BSA reduced the (unexplained) inter-individual variability (IIV) of this parameter from $29.6 \%$ to $24.1 \%$. Intrapatient variability (IOV) was $25.7 \%$. As demonstrated in Figure 2, the elimination of PEGasparaginase was not linear. Several models with time dependent CL were evaluated. ${ }^{20}$ The structural model without the effect of covariates best described the data with a CL of $0.075 \mathrm{~L} /$ day $/ \mathrm{m}^{2}$ for the first 12.9 days after a dose, increasing with 0.079 $\mathrm{L} /$ day $/ \mathrm{m}^{2}$ per day thereafter. Thus, during the first 12.9 days, the half-life of PEGasparaginase was 8.5 days and, thereafter, decreased to 4.1 days after 1 day, and 2.7 days after 2 days.

\section{Covariate analysis}

The univariate analysis resulted in 16 covariates significantly correlated with PEGasparaginase CL (Table 3). In 


\begin{tabular}{|c|c|}
\hline Protocol 1A & Protocol 1B \\
\hline 144 & 142 \\
\hline $\begin{array}{l}\text { asparaginase } \\
\text { levels }\end{array}$ & $\begin{array}{c}\text { asparaginase } \\
\text { levels }\end{array}$ \\
\hline - Prednisone & - Cyclophosphamide \\
\hline - Vincristine & - Cytarabine \\
\hline - Daunorubicin & -6-Mercaptopurine \\
\hline
\end{tabular}

$\rightarrow \quad \frac{\text { Protocol M }}{69}$
$\begin{gathered}\text { asparaginase } \\ \text { levels }\end{gathered}$
- Methotrexate
- 6-Mercaptopurine
$\rightarrow$ MR Intensification $\rightarrow$ MR Maintenance 168
asparaginase levels

$\begin{array}{ll}\text { TEL/AML1 negative } & \\ \text { 139 levels } & \\ \text { - Dexamethasone } & \text { - Dexamethasone } \\ \text { - Vincristine } & \text { - Vincristine } \\ \text { - Doxorubicin } & \text { - Methotrexate } \\ \text { - 6-Mercaptopurine } & \text { - 6-Mercaptopurine }\end{array}$

$\underline{\text { TEL/AML1 positive }}$

29 levels

- Dexamethasone

- Vincristine

- Methotrexate

- 6-Mercaptopurine

$\rightarrow$ SR protocol IV

38 levels

- Dexamethasone

- Vincristine

Figure 1. Concomitant chemotherapy. Types of chemotherapy for each treatment phase. MR: medium risk; SR: standard risk.

the stepwise forward inclusion procedure, the presence of an infection was first added to the structural model, followed by the treatment phase. The CL increased by $38 \%$ when a patient had an infection. In comparison with the treatment phase $1 \mathrm{~A}$, which was used as a reference, $\mathrm{CL}$ was lower during protocol M, MR intensification and maintenance, and SR protocol IV. This association was independent of the presence of an infection. The CL in phase $1 \mathrm{~B}$ was equal to $1 \mathrm{~A}$. Only two patients were treated as high risk, so the association between the HR blocks and PEGasparaginase CL could not be estimated reliably and was fixed to one. The addition of ICU admission to the model (besides the infection and treatment phase), did not improve the fit of the model to the data, neither did the addition of other covariates improve the fit.

The evaluation of the effect of specific chemotherapeutic agents on CL was difficult because blood concentrations of those agents were not measured and many chemotherapeutics agents were administrated concomitantly. Therefore, it was assumed that a possible effect of the agent on $\mathrm{CL}$ was present during the entire treatment phase. As a result, the treatment phase and the administration of a chemotherapeutic agent were highly correlated.

However, as shown in Figure 1, the intensification phase of MR patients with a TEL/AML1 translocation did not contain doxorubicin but these patients were treated with methotrexate additional to dexamethasone, vincristine, 6-mercaptopurine and PEGasparaginase. The other MR patients first received four times doxorubicin in 12 weeks, followed by methotrexate. Although not significant in the univariate analysis, based on clinical grounds, both doxorubicin and methotrexate were evaluated in the multivariate analysis as the effect of these drugs could be analyzed independently of the treatment phase. In the univariate analysis, CL appeared $11 \%$ lower during $M R$ intensification and $19 \%$ lower during $M R$ maintenance compared to protocol $1 \mathrm{~A}$.

The separate addition of the agents to the model
Table 1. Sample characteristics.

\begin{tabular}{lcc} 
& $\begin{array}{c}\text { Index dataset } \\
n=92\end{array}$ & $\begin{array}{c}\text { Validation dataset } \\
n=28\end{array}$ \\
Total number of samples & 816 & 405 \\
Number of levels per patient, & $3(2-12)(1-38)$ & $6(2-33)(1-39)$ \\
median (IQR) (range) & & \\
\hline Sample time (\%) & \\
0-7 days & $371(45 \%)$ & $181(45 \%)$ \\
$8-14$ days & $342(42 \%)$ & $187(46 \%)$ \\
$>14$ days & $103(13 \%)$ & $37(9 \%)$ \\
Number of levels per treatment phase & \\
1A & $144(18 \%)$ & $68(17 \%)$ \\
1B & $142(17 \%)$ & $39(10 \%)$ \\
M & $69(8 \%)$ & $19(5 \%)$ \\
MR intensification & $168(21 \%)$ & $94(23 \%)$ \\
MR maintenance & $250(30 \%)$ & $177(43 \%)$ \\
SR protocol IV & $38(5 \%)$ & $8(2 \%)$ \\
HR blocks & $5(1 \%)$ & - \\
\hline
\end{tabular}

IQR: interquartile range; SR: standard risk; MR: medium risk; HR: high risk.

improved the fit of the model (doxorubicin higher CL, methotrexate lower CL). However, in the backward elimination, both drugs did not influence the PEGasparaginase CL significantly and were, therefore, excluded from the final model.

Thus, the final popPK model included, on top of BSA, the presence of an infection and treatment phase, as covariates significantly associated with the CL of PEGasparaginase. Inclusion of these parameters reduced the IIV of CL from $24.1 \%$ to $19.7 \%$ (18\% reduction), and IOV from $25.7 \%$ to $23.6 \%$ (8\% reduction) (Table 2 ). In this final model, CL was $0.084 \mathrm{~L} /$ day $/ \mathrm{m}^{2}$ for 12.7 days, increasing with $0.082 \mathrm{~L} /$ day $/ \mathrm{m}^{2} /$ day thereafter. 
Table 2. Pharmacokinetic models.

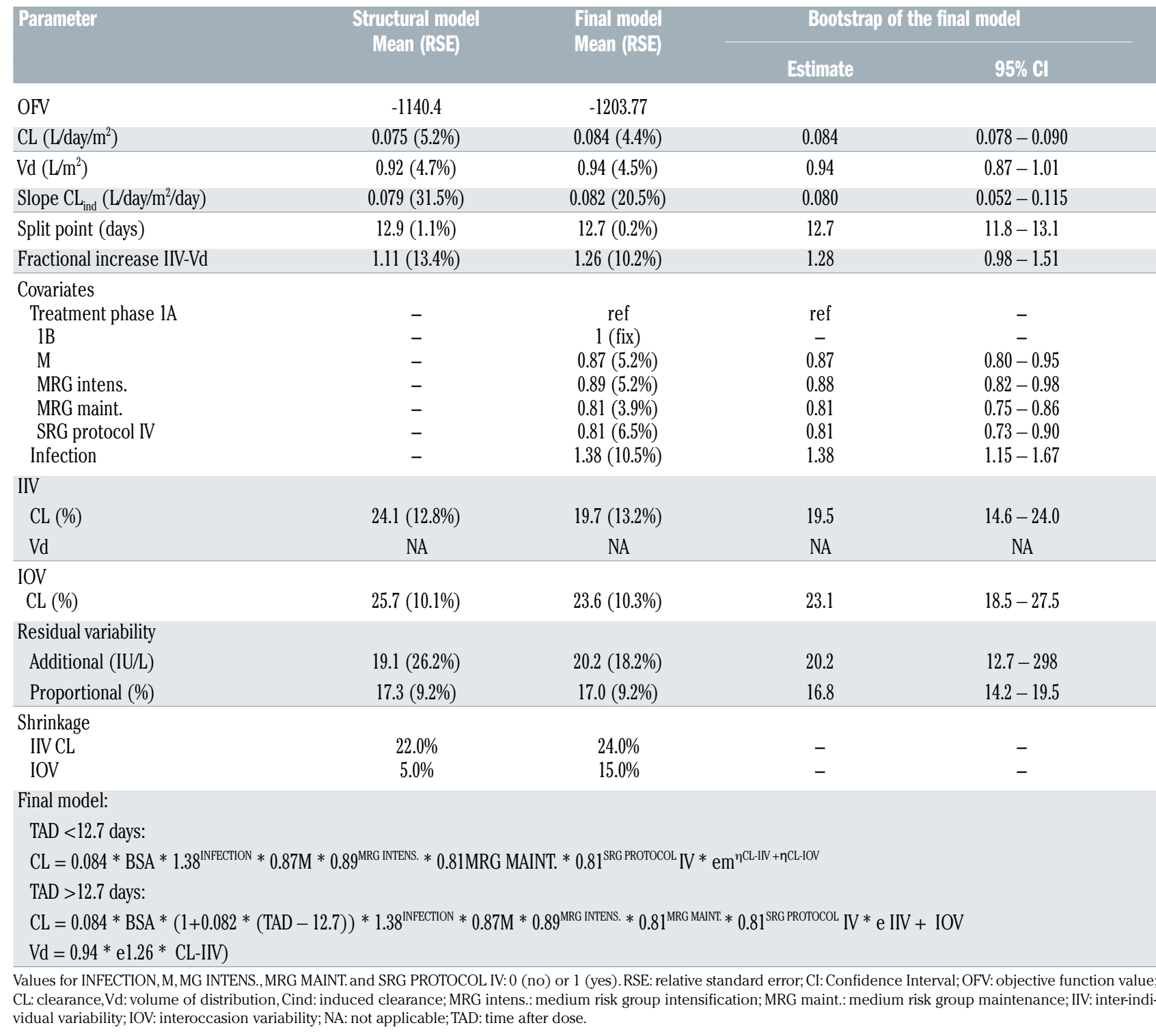

\section{Goodness of fit and model validation}

The parameter estimates of the final model were precise with regards to the relative standard errors, and the shrinkage values were acceptable (Table 2). The goodness of fit plots showed an even distribution of the population predictions and individual predictions around the line of unity (Online Supplementary Figure S1). The conditional weighted residuals, plotted against the time after dose, were also evenly distributed with a mean of zero, and no trend was found. The bootstrap analysis showed that the bootstrap estimates were consistent with the parameter estimates of the final model and the 95\% Confidence Intervals (CI) were accurate (Table 2). The visual predictive check of the index dataset is shown in Figure 3. Both the median estimates and the $95 \% \mathrm{CI}$ are within the simulated predicted values. Thus, the final model is accurate in predicting the PEGasparaginase popPK.

As an external validation, the final model derived from the index dataset was used to predict the asparaginase activity levels of the independent validation dataset. The goodness of fit plots and VPC of the validation dataset also show that the final model is adequate to describe the PEGasparaginase PK (Online Supplementary Figures S1 and $S 3$ ).

\section{Clinical implications}

Table 4 shows several dosing regimens for PEGasparaginase (loading dose and maintenance dose) during induction and MRG intensification. Targeting a trough PEGasparaginase activity level of $100 \mathrm{IU} / \mathrm{L}$, the loading dose recommended during induction is $800 \mathrm{IU} / \mathrm{m}^{2}$ followed by a biweekly maintenance dose of $600 \mathrm{IU} / \mathrm{m}^{2}$. Lower doses should be administered due to decreased CL for PEGasparaginase treatment during intensification, with doses being $600 \mathrm{IU} / \mathrm{m}^{2}$ and $400 \mathrm{IU} / \mathrm{m}^{2}$, respectively, to target $100 \mathrm{IU} / \mathrm{L}$.

Based on the half-life during the first 13 days, the steady state is estimated to be reached after two doses, after which the dose can be adjusted based on trough or week asparaginase activity levels targeting trough levels between 100-250 IU/L or 250-400 IU/L (Table 5). If an 
Table 3. Univariate covariate analysis.

\begin{tabular}{|c|c|c|c|}
\hline Covariate & Effect: $\Theta(95 \%$ Cl) & $\triangle 0 F V$ & Included after backward elimination? \\
\hline $\begin{array}{l}\text { Anti-native } E \text {. coli asparaginase } \\
\text { antibodies (extinction values) }\end{array}$ & $0.05(-0.01-0.11)$ & -12.9 & No \\
\hline $\begin{array}{l}\text { Anti-PEGasparaginase } \\
\text { antibodies (extinction values) }\end{array}$ & $0.04(-0.01-0.10)$ & -7.3 & No \\
\hline Creatinine & $-0.21(-0.36--0.07)$ & -17.0 & No \\
\hline Leukocytes & $-0.09(-0.13-0.05)$ & -22.9 & No \\
\hline ICU admission & $1.61(1.55-1.67)$ & -31.0 & No \\
\hline Infection & $1.45(1.11-1.79)$ & -52.5 & Yes \\
\hline \multicolumn{4}{|l|}{ Treatment phase } \\
\hline $1 \mathrm{~A}$ & ref & -42.3 & Yes \\
\hline 1B & 1 (fix) & & \\
\hline M & $0.86(0.78-0.94)$ & & \\
\hline MRG intens. & $0.86(0.77-0.95)$ & & \\
\hline MRG maint. & $0.78(0.71-0.85)$ & & \\
\hline SRG protocol IV & $0.78(0.67-0.88)$ & & \\
\hline \multicolumn{4}{|l|}{ Other chemotherapy } \\
\hline Prednisone & $1.11(0.97-1.25)$ & -5.4 & No \\
\hline Vincristine & $0.89(0.82-0.96)$ & -12.2 & No \\
\hline Daunorubicine & $1.11(0.97-1.25)$ & -5.4 & No \\
\hline 6-Mercaptopurine & $0.91(0.84-0.99)$ & -6.8 & No \\
\hline Cyclophosphamide & $1.13(0.98-1.22)$ & -5.3 & No \\
\hline Cytarabine & $1.13(0.93-1.33)$ & -5.3 & No \\
\hline Methotrexate & $0.85(0.80-0.91)$ & -26.2 & No \\
\hline Dexamethasone & $0.85(0.78-0.91)$ & -27.7 & No \\
\hline Doxorubicin & $1.01(0.90-1.12)$ & -0.06 & No \\
\hline
\end{tabular}

${ }^{*}$ In order to evaluate any chemotherapy-related effects on clearance on top of the treatment phase, all chemotherapy agents were first included in the multivariate analysis. Only inclusion of doxorubicin and methotrexate resulted in a significant improvement of the model (OFV -6.0, mean effect (RSE): 1.24 (6\%) and OFV -10.3, mean effect (RSE): 0.88 $(5 \%)$, respectively). CI: Confidence Interval; OFV: objective function value; ICU: intensive care unit, MRG intens.: medium risk intensification; MRG maint.: medium risk maintenance phase. Covariates significantly influenced the clearance in the univariate analysis when the OFV decreased with $>3.84(P<0.05)$.

infection occurs, it is advised to increase the dose with $38 \%$, if clinically possible.

\section{Discussion}

In this study, the popPK of PEGasparaginase were investigated in order to identify patient and clinical characteristics associated with the CL of PEGasparaginase. Furthermore, the popPK model was used to develop a dosing guideline for the drug. It was shown that the $\mathrm{CL}$ is constant during the first 13 days after administration and increases thereafter. In addition, the CL was higher during induction, and during an infection.

During development of the structural model, neither first- and zero-order, nor Michaelis Menten elimination described the concentration-time profile of PEGasparaginase adequately. This has also been reported by Hempel et al..$^{17}$ and Würthwein et al..$^{20}$ In the present study, similar to Würthwein et al., the time dependency of $\mathrm{CL}$ proved to be described most adequately with a split model, showing that CL increases substantially after a period of approximately 13 days. This induced CL could be explained by the hydrolysis of the PEG moiety from the PEGasparaginase molecule, resulting in native E. coli asparaginase with a linker attached, originally connecting PEG to asparaginase. Thus, CL will increase to a value more or less comparable with that of native E. coli asparaginase, which has a half-life of 1.3 days ${ }^{16}$ after several days.

Several associations between covariates and the PEGasparaginase CL were identified. The metabolism of PEGasparaginase appears to depend on the treatment phase and is influenced by the presence of an infection. Native E. coli asparaginase is eliminated mainly by macrophages of the mononuclear phagocyte system (MPS) in the bone marrow, spleen and liver. ${ }^{21}$ Although the mechanism of elimination of PEGasparaginase has not been studied, it is likely that this also holds for the PEGylated formulation as other PEGylated drugs are also eliminated by cells of the MPS. ${ }^{22}$

$\mathrm{CL}$ was the highest during protocol $1 \mathrm{~A}$ containing vincristine, prednisone and daunorubicin. MR intensification, maintenance and SR protocol IV had lower CL and all contained vincristine as well, so an effect of vincristine is unlikely. Especially as the CL during MR maintenance was lower than MR intensification, either doxorubicin and/or methotrexate probably influence the PEGasparaginase CL. The fact that the CL was the highest in induction might be caused by the other anthracycline, daunorubicin, which was administered weekly, whereas doxorubicin, was administered once every 3 weeks during intensification.

Besides the administration of specific chemotherapeutics, other mechanisms may explain the differences in $\mathrm{CL}$ 


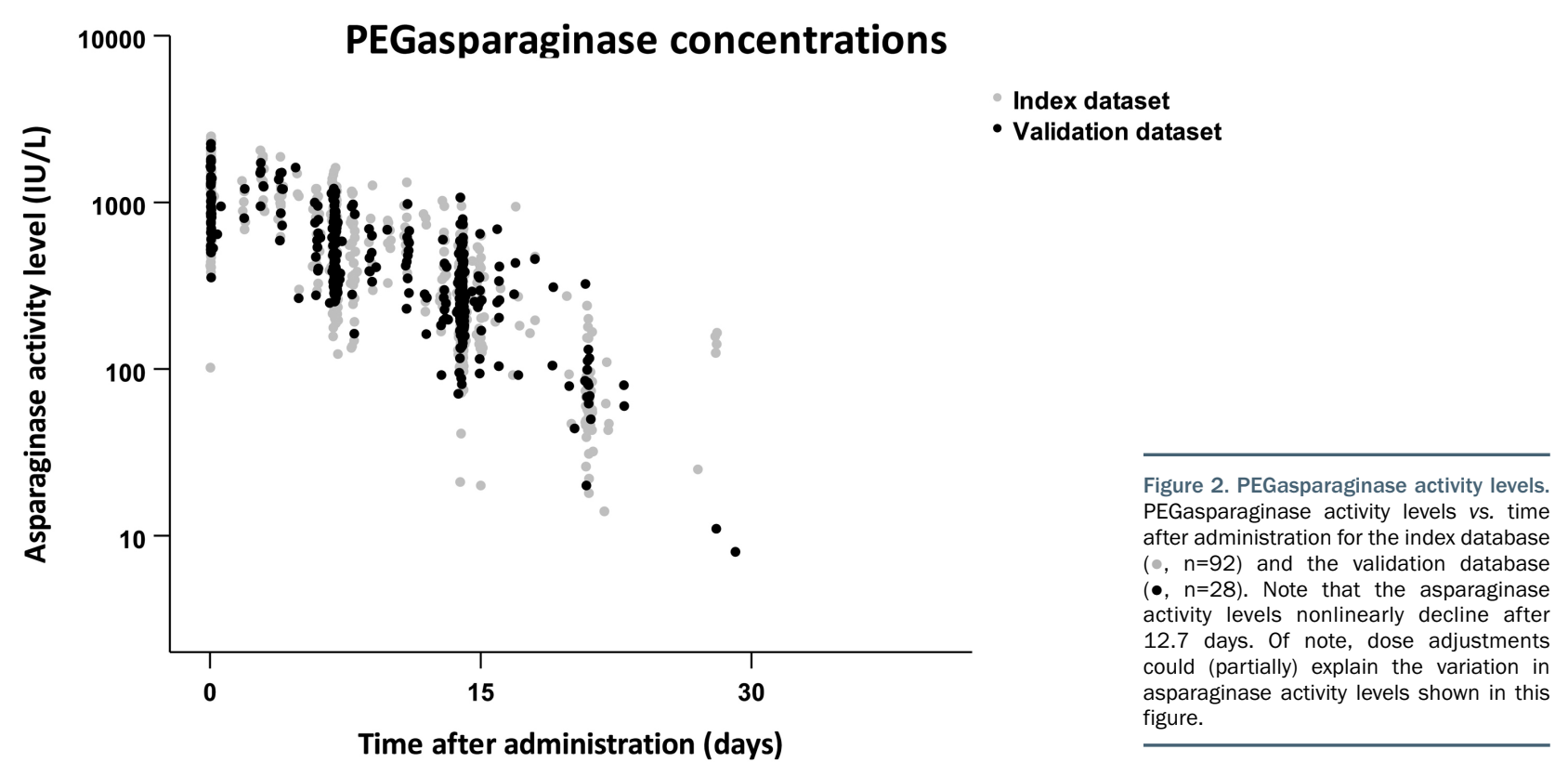

Table 4. Dosing guideline, starting dose.

\begin{tabular}{lccc} 
& Target trough asparaginase level & Moading dose & Maintenance dose \\
Protocol 1A (induction) & $100 \mathrm{IU} / \mathrm{L}$ & $800 \mathrm{IU} / \mathrm{m}^{2}$ & $600 \mathrm{IU} / \mathrm{m}^{2}$ \\
& $250 \mathrm{IU} / \mathrm{L}$ & $1,800 \mathrm{IU} / \mathrm{m}^{2}$ & $1,400 \mathrm{IU} / \mathrm{m}^{2}$ \\
& $350 \mathrm{IU} / \mathrm{L}$ & $2,200 \mathrm{IU} / \mathrm{m}^{2}$ & $1,600 \mathrm{IU} / \mathrm{m}^{2}$ \\
Medium risk intensification & $100 \mathrm{IU} / \mathrm{L}$ & $600 \mathrm{IU} / \mathrm{m}^{2}$ & $400 \mathrm{IU} / \mathrm{m}^{2}$ \\
& $250 \mathrm{IU} / \mathrm{L}$ & $1,500 \mathrm{IU} / \mathrm{m}^{2}$ & $1,000 \mathrm{IUU} / \mathrm{m}^{2}$ \\
& $350 \mathrm{IU} / \mathrm{L}$ & $1,800 \mathrm{IU} / \mathrm{m}^{2}$ & $1,800 \mathrm{IU} / \mathrm{m}^{2}$ \\
\hline
\end{tabular}

Table 5. Dosing guideline, dose adjustments.

\begin{tabular}{|c|c|c|c|c|c|}
\hline \multirow[b]{2}{*}{ Week level } & \multicolumn{2}{|c|}{ arget trough level: 100-250 IU/L } & \multicolumn{3}{|c|}{ Target trough level: $250-400$ IU/L } \\
\hline & Trough level & Dose adjustment & Week level & Trough level & Dose adjustment \\
\hline $50-100 \mathrm{IU} / \mathrm{L}$ & $25-50 \mathrm{IU} / \mathrm{L}$ & $400 \%$ & $100-200 \mathrm{IU} / \mathrm{L}$ & $50-100 \mathrm{IU} / \mathrm{L}$ & $400 \%$ \\
\hline $100-250 \mathrm{IU} / \mathrm{L}$ & 50-75 IU/L & $300 \%$ & 200-300 IU/L & $100-150 \mathrm{IU} / \mathrm{L}$ & $300 \%$ \\
\hline $150-200 \mathrm{IU} / \mathrm{L}$ & 75-100 IU/L & $200 \%$ & $300-250 \mathrm{IU} / \mathrm{L}$ & $150-200 \mathrm{IU} / \mathrm{L}$ & $200 \%$ \\
\hline $200-450 \mathrm{IU} / \mathrm{L}$ & 100-250 IU/L & $100 \%$ & $350-450 \mathrm{IU} / \mathrm{L}$ & $200-250 \mathrm{IU} / \mathrm{L}$ & $175 \%$ \\
\hline $450-550 \mathrm{IU} / \mathrm{L}$ & 250-300 IU/L & $60 \%$ & $450-700 \mathrm{IU} / \mathrm{L}$ & $250-400 \mathrm{IU} / \mathrm{L}$ & $100 \%$ \\
\hline $550-750 \mathrm{IU} / \mathrm{L}$ & 300-400 IU/L & $50 \%$ & 700-900 IU/L & 400-500 IU/L & $70 \%$ \\
\hline $750-1,100 \mathrm{IU} / \mathrm{L}$ & 400-600 IU/L & $40 \%$ & $900-1,100 \mathrm{IU} / \mathrm{L}$ & $500-600 \mathrm{IU} / \mathrm{L}$ & $60 \%$ \\
\hline $1100-1,500 \mathrm{IU} / \mathrm{L}$ & $600-800 \mathrm{IU} / \mathrm{L}$ & $25 \%$ & $1100-1,250 \mathrm{IU} / \mathrm{L}$ & $600-700 \mathrm{IU} / \mathrm{L}$ & $50 \%$ \\
\hline $1500-1,800 \mathrm{IU} / \mathrm{L}$ & $800-1,000 \mathrm{IU} / \mathrm{L}$ & $20 \%$ & $1250-1,800 \mathrm{IU} / \mathrm{L}$ & $700-1,000 \mathrm{IU} / \mathrm{L}$ & $35 \%$ \\
\hline
\end{tabular}

The dose adjustments apply for biweekly administration of PEGasparaginase during steady state. The doses may be adjusted based 1 week (7 days) or trough (14 days) after administration targeting at trough asparaginase activity levels of 100-250 IU/L or 250-400 IU/L.

between the treatment phases. It has to be taken into account that the physical condition of the patient alters during ALL treatment: during protocol 1A (and also 1B), the tumor load is higher and therapy is more intense compared to protocol M, MR intensification and maintenance, and SR protocol IV, which all had a lower CL. The MPS plays a role in tumor lysis, ${ }^{23,24}$ which may explain why the $\mathrm{CL}$ is higher during this treatment phase as well, although the asparaginase treatment starts at day 12 of the treatment protocol.

Strikingly, the occurrence of an infection increases the $\mathrm{CL}$ by $38 \%$, independent of the treatment phase and BSA, probably because of the activation of the MPS, which is responsible for the CL of asparaginase. This means that the PEGasparaginase activity levels will be $38 \%$ lower during an infection and that the PEGasparaginase dose should be increased by this percentage to obtain similar levels. Evidently, this should first be validated in clinical practice, taking into account the clinical situation of the patient.

BSA was included in the structural model, showing that the CL of PEGasparaginase increases with BSA, which is in line with the findings of Hempel et al. ${ }^{17}$ Sassen et al., however, studied the CL of Erwinia asparaginase and 
found the opposite: patients with a lower weight have a higher CL, requiring a higher starting dose of the drug. ${ }^{25}$ Shrey et al. studied the native E. coli asparaginase levels in different age groups and also showed lower asparaginase activity levels in younger patients, suggesting a higher CL in these patients. ${ }^{26}$ Thus, for a PEGylated form of asparaginase, this does not seem to apply, which is also supported by a study of PEGylated interferon $\alpha-2 b$ in children, which also shows that the CL increases with age. ${ }^{27}$
In our analysis, PEGasparaginase CL was not statistically correlated with asparaginase-related toxicity. The correlation between toxicity and asparaginase activity levels was also studied in 382 pediatric patients treated according to the DCOG ALL-11 protocol. In these patients, PEGasparaginase treatment, as in the current cohort, was individualized based on asparaginase activity levels, resulting in much lower trough levels compared to patients who were treated according to the DCOG ALL-

A

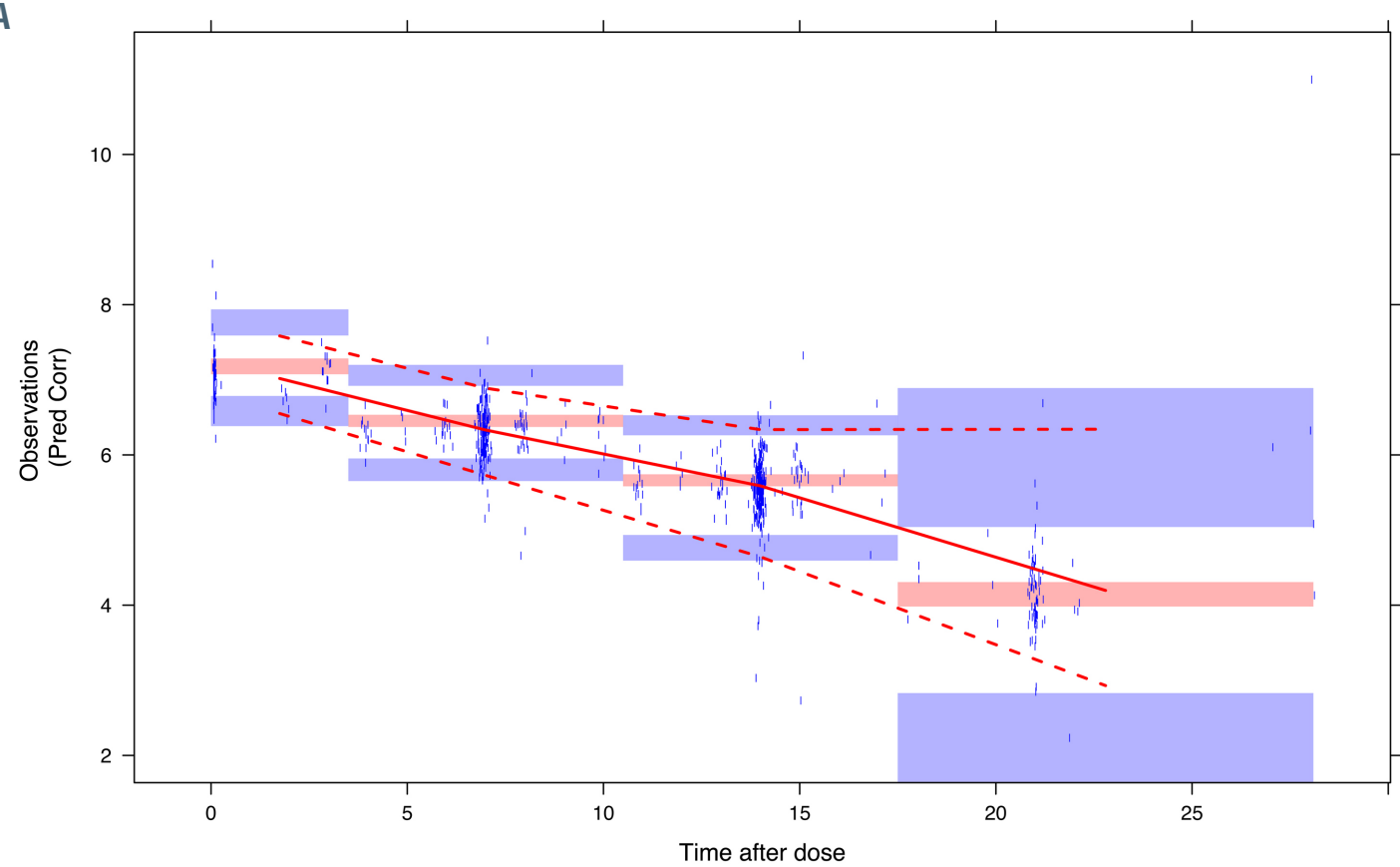

B

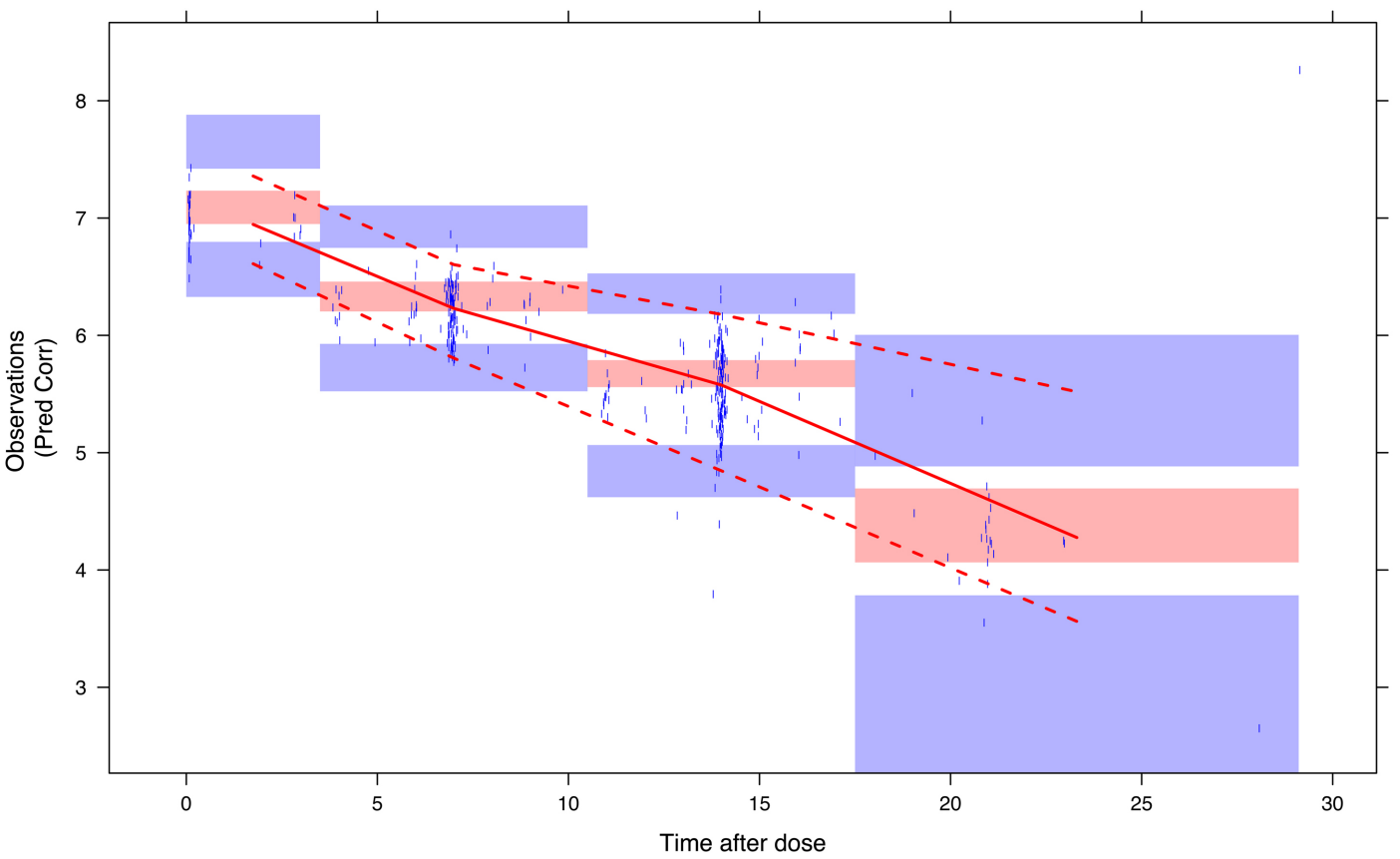

Figure 3. Visual predictive check. (A) and (B) show the visual predictive checks of the index and validation dataset, respectively. The observations and their corresponding median and 95\% Confidence Intervals $(\mathrm{Cl})$ are indicated by the points, and the solid and dashed red lines. In both graphs, these lines fall within the $95 \%$ $\mathrm{Cl}$ of the median and $95 \% \mathrm{Cl}$ (red and blue shaded areas) as obtained by simulation. 
10 protocol, which had a fixed PEGasparaginase dose of $2,500 \mathrm{IU} / \mathrm{m}^{2}$. The asparaginase-related toxicity in both protocols, however, was similar and there was no correlation between asparaginase activity levels. ${ }^{28}$

Based on the popPK model, dosing guidelines were developed taking into account the effect of the treatment phase on PEGasparaginase CL. Next, dose adjustments based on both week and trough levels were suggested targeting 100-250 IU/L or the higher range of 250-400 IU/L. These dosing guidelines, however, should first be validated clinically. In addition, the effect of increasing the dose in case of an infection on PEGasparaginase activity levels should be analyzed.

In conclusion, $18 \%$ of the inter- and $8 \%$ of the intrapatient variability in CL of PEGasparaginase, normalized for BSA, can be explained by i) treatment phase showing a higher CL during induction treatment (protocol 1A) and ii) the occurrence of an infection, which increases the CL. With the popPK model developed in this study,
PEGasparaginase treatment may be individualized further, taking into account these covariates and the dosing guidelines provided.

\section{Disclosures}

$R K$ has non conflicts of interest to declare; RP and IMvdS has received research support and consultancy fees from Jazz Pharmaceuticals and Medac and $R M$ has received travel grants from Shire and Bayer.

\section{Contributions}

$R K$ collected the data, performed the analysis, interpreted the data and wrote the manuscript; $R M$ performed the analysis, interpreted the data and wrote the manuscript; $R P$ and IMvdS designed the research, interpreted the data and wrote the manuscript.

\section{Funding}

This work was supported by the KiKa foundation.

\section{References}

1. Broome JD. L-Asparaginase: discovery and development as a tumor-inhibitory agent. Cancer Treat Rep. 1981;65 Suppl 4:111-114.

2. Bussolati O, Belletti S, Uggeri J, et al. Characterization of apoptotic phenomena induced by treatment with L-asparaginase in NIH3T3 cells. Exp Cell Res. 1995;220(2):283291.

3. Miller HK, Salser JS, Balis ME. Amino acid levels following L-asparagine amidohydrolase (EC.3.5.1.1) therapy. Cancer Res. 1969;29(1):183-187

4. Ahlke E, Nowak-Gottl U, Schulze-Westhoff $\mathrm{P}$, et al. Dose reduction of asparaginase under pharmacokinetic and pharmacodynamic control during induction therapy in children with acute lymphoblastic leukaemia. Br J Haematol. 1997;96(4):675681.

5. Albertsen BK, Schroder H, Ingerslev J, et al. Comparison of intramuscular therapy with Erwinia asparaginase and asparaginase Medac: pharmacokinetics, pharmacodynamics, formation of antibodies and influence on the coagulation system. $\mathrm{Br} \mathrm{J}$ Haematol. 2001;115(4):983-990.

6. Grigoryan RS, Panosyan EH, Seibel NL, et al. Changes of amino acid serum levels in pediatric patients with higher-risk acute lymphoblastic leukemia (CCG-1961). In Vivo. 2004;18(2):107-112.

7. Riccardi R, Holcenberg JS, Glaubiger DL, et al. L-asparaginase pharmacokinetics and asparagine levels in cerebrospinal fluid of rhesus monkeys and humans. Cancer Res. 1981;41(11 Pt 1):4554-4558.

8. Appel IM, Kazemier KM, Boos J, et al. Pharmacokinetic, pharmacodynamic and intracellular effects of PEG-asparaginase in newly diagnosed childhood acute lymphoblastic leukemia: results from a single agent window study. Leukemia. 2008;22(9): 1665-1679.

9. Rizzari C, Zucchetti M, Conter V, et al. L- asparagine depletion and L-asparaginase activity in children with acute lymphoblastic leukemia receiving i.m. or i.v. Erwinia C. or E. coli L-asparaginase as first exposure. Ann Oncol. 2000;11(2):189-193

10. Tsurusawa $M$, Chin M, Iwai $A$, et al. I Asparagine depletion levels and L-asparaginase activity in plasma of children with acute lymphoblastic leukemia under asparaginase treatment. Cancer Chemother Pharmacol. 2004;53(3):204-208.

11. Asselin BL, Whitin JC, Coppola DJ, et al Comparative pharmacokinetic studies of three asparaginase preparations. J Clin Oncol. 1993;11(9):1780-1786.

12. Pieters R, Hunger SP, Boos J, et al. L-asparaginase treatment in acute lymphoblastic leukemia: a focus on Erwinia asparaginase. Cancer. 2011;117(2):238-249.

13. Avramis VI, Sencer S, Periclou AP, et al. A randomized comparison of native Escherichia coli asparaginase and polyethylene glycol conjugated asparaginase for treatment of children with newly diagnosed standard-risk acute lymphoblastic leukemia: a Children's Cancer Group study. Blood. 2002;99(6):1986-1994.

14. Hawkins DS, Park JR, Thomson BG, et al. Asparaginase pharmacokinetics after intensive polyethylene glycol-conjugated Lasparaginase therapy for children with relapsed acute lymphoblastic leukemia. Clin Cancer Res. 2004:10(16):5335-5341.

15. Silverman LB, Gelber RD, Dalton VK, et al. Improved outcome for children with acute lymphoblastic leukemia: results of DanaFarber Consortium Protocol 91-01. Blood. 2001;97(5):1211-1218.

16. Asselin BL. The three asparaginases. Comparative pharmacology and optimal use in childhood leukemia. Adv Exp Med Biol. 1999:457:621-629.

17. Hempel G, Muller HJ, Lanvers-Kaminsky C, et al. A population pharmacokinetic mode for pegylated-asparaginase in children. $\mathrm{Br}$ Haematol. 2010;148(1):119-125.
18. Tong WH, Pieters R, Kaspers GJ, et al. A prospective study on drug monitoring of PEGasparaginase and Erwinia asparaginase and asparaginase antibodies in pediatric acute lymphoblastic leukemia. Blood. 2014; 123(13):2026-2033.

19. Mosteller RD. Simplified calculation of body-surface area. N Engl J Med. 1987; 317(17):1098.

20. Wurthwein G, Lanvers-Kaminsky C Hempel G, et al. Population pharmacokinetics to model the time-varying clearance of the PEGylated Aaparaginase oncaspar(R) in children with acute lymphoblastic leukemia. Eur J Drug Metab Pharmacokinet. 2017;42(6):955-963.

21. van der Meer LT, Terry SY, van Ingen Schenau DS, et al. In vivo imaging of antileukemic drug asparaginase reveals a rapid macrophage-mediated clearance from the bone marrow. J Nucl Med. 2017; 58(2):214-220

22. Yang Q, Lai SK. Anti-PEG immunity: emergence, characteristics, and unaddressed questions. Wiley Interdiscip Rev Nanomed Nanobiotechnol. 2015;7(5):655-677.

23. Adams DO. Tumor cell recognition and destruction by activated macrophages: effector and regulatory mechanisms. Dordrecht: Springer, 1985.

24. Becker S. Functions of the human mononuclear phagocyte system. Adv Drug Deliver Rev. 1988;2(1-29).

25. Sassen SD, Mathot RA, Pieters R, et al. Population pharmacokinetics of intravenous Erwinia asparaginase in pediatric acute lymphoblastic leukemia patients. Haematologica. 2017;102(3):552-561.

26. Schrey D, Borghorst S, Lanvers-Kaminsky $\mathrm{C}$, et al. Therapeutic drug monitoring of asparaginase in the ALL-BFM 2000 protocol between 2000 and 2007. Pediatr Blood Cancer. 2010;54(7):952-958

27. Xu C, Gupta S, Krishna G, et al. Population pharmacokinetics of peginterferon alfa- $2 b$ in pediatric patients with chronic hepatitis C. 